\title{
Better, faster, cheaper: Recent SCHOTT ZERODUR facilities are game-changing for high performance lightweight mirrors
}

Thomas Westerhoff, Tony Hull, Janina Krieg

Thomas Westerhoff, Tony Hull, Janina Krieg, "Better, faster, cheaper: Recent SCHOTT ZERODUR facilities are game-changing for high performance lightweight mirrors," Proc. SPIE 11852, International Conference on Space Optics - ICSO 2020, 118524R (11 June 2021); doi: 10.1117/12.2599807

SPIE Event: International Conference on Space Optics - ICSO 2021, 2021, Online Only 


\section{International Conference on Space Optics-ICSO 2020}

Virtual Conference

30 March-2 April 2021

Edited by Bruno Cugny, Zoran Sodnik, and Nikos Karafolas
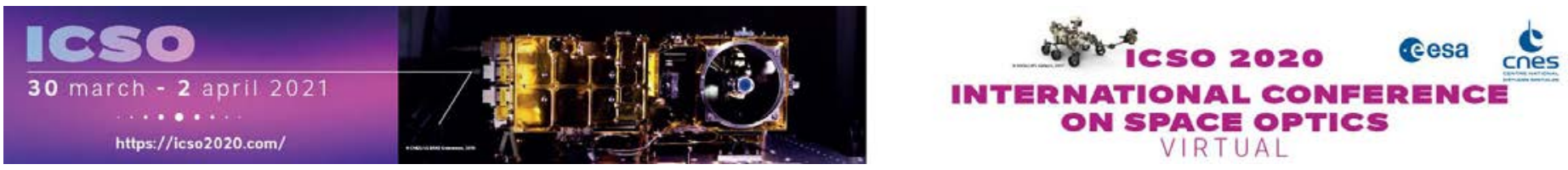

\section{Better, faster, cheaper: Recent SCHOTT ZERODUR facilities are game-changing for high performance lightweight mirrors}

\section{Cesa isoporacedings ecnes}




\title{
Better, faster, cheaper: Recent SCHOTT ZERODUR ${ }^{\circledR}$ facilities are game-changing for high performance lightweight mirrors WESTERHOFF Thomas ${ }^{1}$, HULL Tony ${ }^{2}$, KRIEG Janina ${ }^{1}$ \\ ${ }^{1}$ SCHOTT AG, Mainz, DE \\ ${ }^{2}$ Department of Physics \& Astronomy, University of New Mexico US
}

\begin{abstract}
"Better" has always been a factor in selecting materials for spaceborne use. Increasingly, cost and delivery schedule are discriminating parameters, especially where constellations of satellites are to be implemented. Recent facilitization at SCHOTT guarantees not only rapid access to lightweight ZERODUR ${ }^{\circledR}$ mirror blanks, even in the era of needing approaching $10^{3}$ mirrors for the VLT, but also advanced multi-axis fabrication and metrology equipment enables generated optical surfaces with low form error and low sub-surface damage. This in turn may significantly reduce the time of optical fabrication and assembly. We see this as a vital step toward realizing Design-to-Cost paradigms, faster and cheaper high performance optical assemblies.
\end{abstract}

\section{INTRODUCTION}

ZERODUR ${ }^{\circledR}$ was developed by SCHOTT 53 years ago in support of ground astronomy, where optical mirrors require great dimensional stability over extended temperature excursions. This established a material, unchanged in formulation over this period, providing both superb abilities to be smoothly polished and provide extreme thermal stability [1]. ZERODUR ${ }^{\circledR}$ produced in 1968 is stable and that produced today is rigorously controlled to be identical. Presently available in sizes to 4 plus meters in diameter, ZERODUR ${ }^{\circledR}$ satisfies most present and future telescope needs, both for ground astronomy, and for much of this period, spaceborne missions.

ZERODUR ${ }^{\circledR}$ soon became the thermal stability standard for other precision technologies, including the semiconductor and laser gyro industries, this in turn ensuring robust continuous production and characterization since inception. The material has been extensively characterized by SCHOTT and others, including NASA's recent thermal-vacuum test of a $1.2 \mathrm{~m}$ aggressively lightweighted mirror at the XRCF facility [2]. ZERODUR ${ }^{\circledR}$ has successfully flown on well over 40 missions [3], and over the last decade is available from SCHOTT with significant mass relief via backside machining of isogrid forms [4]. Due to the broad industrial applications for ZERODUR ${ }^{\circledR}$, significant inventories of the material are in stores, providing attractive delivery schedules of this material.

ZERODUR ${ }^{\circledR}$ mirror blanks are being provided for nearly all the mirrors of European Southern Observatory's (ESO's) Extremely Large Telescope (ELT), the world's biggest "eye on the sky" $[5,6]$. SCHOTT has responded with manufacturing facilities, capacity and flow plan to deliver over $10^{3}$ segment blanks for optical processing, while maintaining capacity for advanced mirrors, including those lightweighted for space, and significant industrial demand.

We will address both the process, facilities and capabilities to make lightweight ZERODUR ${ }^{\circledR}$ substrates, as well as recent powerful augmentations to SCHOTT's process to increase the efficiency of producing an optically finished high performance ZERODUR ${ }^{\circledR}$ mirror. 


\section{ZERODUR ${ }^{\circledR}$ PROCESS FLOW FROM CASTING THROUGH MACHINING}

\section{Established process flow:}

Figure 1 expresses the process to make ZERODUR ${ }^{\circledR}$. It is important to emphasize that ZERODUR ${ }^{\circledR}$ mirror blank material cast in a boule, not made by deposition, joining, deposition or infusion. This ensures uncommon homogeneity of all physical properties throughout the blank. In the case of the $4.26 \mathrm{~m}$ diameter Daniel K. Inouye Solar Telescope (DKIST), homogeneity of the coefficient of thermal expansion (CTE) of ZERODUR ${ }^{\circledR}$ is established to be better than \pm 5 parts per billion (ppb) [7,8], near the threshold of metrology of CTE

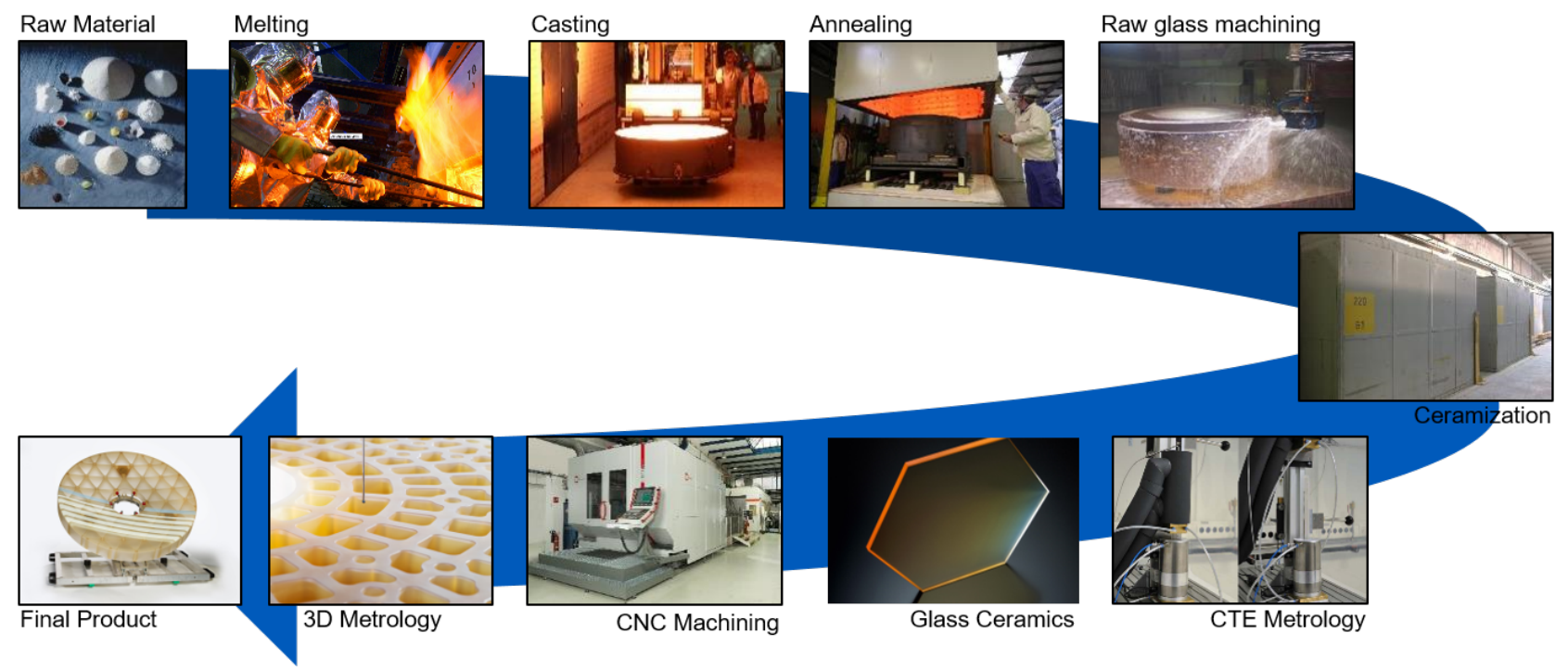

Figure 1: The well-established process flow for ZERODUR ${ }^{\circledR}$ produces mirror substrates that are extremely homogeneous in properties, including CTE, and simultaneously extremely low CTEs. A decade ago, SCHOTT established and offers a high degree of lightweighting of the mirror blanks via efficient and deterministic machining processes. The example at the lower left is $1.2 \mathrm{~m}$ in diameter, $88 \%$ lightweighted, and produces a first eigenfrequency of $210 \mathrm{~Hz}$.

\section{Alternate processes to make lightweight mirror substrates:}

A critical attribute of material relevance is the ability of the material to be processed into a highly smooth optical finish.

1. While single point diamond turning can provide a specular surface on bare aluminum mirrors to be used in the infrared, and sometimes down to $500 \mathrm{~nm}$ wavelength, eventually as wavelengths go shorter, the periodic errors associated with the diamond turning process introduce unacceptable scatter. Some groups have developed ways to post-polish diamond turned aluminum, though scatter from the surface is still an issue as wavelengths get shorter.

2. Traditionally bare aluminum, bare beryllium and bare SiC do not polish well. While as evidenced by JWST, bare beryllium can be polished for applications down to wavelengths of the order of 500nm wavelength, though special technology is needed. Optically finishing either bare beryllium or bare aluminum introduces concerns caused by the microyield, microcreep and anisotropy properties of these materials. These attributes are not the concern for glasses, glass ceramics or $\mathrm{SiC}$ provided the mirror blank is made of a single piece of material.

3. Since bare beryllium, bare aluminum and bare $\mathrm{SiC}$ are challenging to polish directly providing a low scatter surface in the visible wavelengths, often these materials are clad with a material that does polish well without inducing optical scatter, a significant noise cause in an optical system. In the case of beryllium and aluminum, 
autocatalytic electroless nickel is often used, and this may be polished to a low microroughness surface of $1 \mathrm{~nm}$ rms or better at high spatial frequencies.

4. Nevertheless, this cladding induces both a stress on the substrate, and also a bimetallic effect as the temperature is changed. To be successful as a stable substrate, an electroless nickel must meet any of three conditions:

a. The shell be exactly the same CTE as the substrate

b. The shell be infinitesimally thin

c. The electroless nickel shell must exactly match the neutral axis of the Al or Be substrate. This is difficult at all spatial frequencies on a lightweighted mirror.

5. While attempts are made to do all $4 \mathrm{a}, \mathrm{b}, \mathrm{c}$, in practice none can be done perfectly. The budget for thermal stability of such mirrors must carry large terms for the bimetallic moment. SiC mirrors to be used at visible and near infrared wavelengths also must be clad with another material. Several solutions are tried including CVD SiC, Silicon, Glass and nanolaminates. Unless the mirror is also made by the CVD process, and typically it is not, the mirror designer must consider and budget for a potential thermal response difference in CVD SiC due to its different structure than the underlying material.

6. With any cladding, the same three criteria for electroless nickel on $\mathrm{Al}$ or Be must be considered.

7. With any cladding, as it is applied thin, this introduces effort to accurately prepare the aspheric form both before and after cladding. This introduces risk of "polishing through" the cladding. When polishing through happens, the process must go back to the generation stage to machine off the cladding and then reclad.

8. ZERODUR ${ }^{\circledR}$ may be polished to an exceptionally smooth surface without cladding.

While our discussion is focused on the material attributes shared by all traditional substrate mirror materials for spaceborne telescopes, we expand on some of the differences the designer must recognize as various materials are considered for mirrors in such a telescope. Table 1 summarizes some of the similarities and differences we are considering.

Table 1. Material Properties Comparison I: Glass ceramics, glass, cordierites and metals. Bold designates favored. Many mirror materials cannot be made into large substrates without joining operations. Joining may consist of bonding, fusing and even brazing. In several ways this introduces degradation of homogeneity, both with assembled parts each with slightly different characteristics, and the nature of the bonding or brazing. An example is the technology demonstration mirror acquired for NASA's Terrestrial Planet Finder Mission. In this case, a 1.8m unobscured mirror was to be fabricated by fusing 9 pieces of ULE [9]

\begin{tabular}{|c|c|c|c|c|c|c|c|}
\hline $\begin{array}{l}\text { Attribute / } \\
\text { Material }\end{array}$ & $\begin{array}{c}\text { Maximum } \\
\text { monolithic diameter }\end{array}$ & Optical surfacing & Breakage Strength & $\begin{array}{l}\text { Micro- } \\
\text { yield }\end{array}$ & $\begin{array}{l}\text { Micro- } \\
\text { creep }\end{array}$ & Anisotropy & $\begin{array}{l}\text { Radiation } \\
\text { compaction }\end{array}$ \\
\hline ZERODUR* & $4.3 \mathrm{~m}$ & Superb & $\begin{array}{c}\text { Sufficient / } \\
\text { published w. tight } \sigma\end{array}$ & No & No & No & $\begin{array}{c}\text { Negligible for } \\
\text { most orbits }\end{array}$ \\
\hline ULE & $\sim 1.5 \mathrm{~m} /$ fuse larger & Good & Limited data & No & No & No & $\begin{array}{c}\text { Negligible for } \\
\text { most orbits }\end{array}$ \\
\hline Fused Silica & $\sim 1.5 \mathrm{~m}$ & Superb & Limited data & No & No & No & $\begin{array}{c}\text { Negligible for } \\
\text { most orbits }\end{array}$ \\
\hline Cordierite 720 & $\sim 1.5 \mathrm{~m}$ & Good & Sufficient & No & No & No & Low \\
\hline $\mathrm{SiC}$ & $\sim 1.5 \mathrm{~m}$ / fuse larger & Must be clad & Sufficient & No & No & Yes, if clad & Low \\
\hline Al & $\sim 0.4 \mathrm{~m}$ & $\begin{array}{c}\text { EN clad for } \lambda< \\
500 \mathrm{~nm}\end{array}$ & Sufficient & Yes & Yes & Yes & Low \\
\hline $\mathrm{Be}$ & $\sim 1.6 \mathrm{~m}$ & $\begin{array}{c}\text { EN clad for } \lambda< \\
500 \mathrm{~nm}\end{array}$ & Sufficient & Yes & Yes & Yes & Low \\
\hline
\end{tabular}

With a mirror needing to provide stable performance at even the nanometer level, of consideration during choice of design and material selection is the quality and completeness of material data present to make an informed design. 


\section{Consideration of Ionizing Radiation:}

For some materials ionizing radiation can induce material compaction, capable of changing both the radius of curvature and irregularity of the mirror surface over time. This is of less concern for mirrors embedded deeply in a telescope, and for normal earth orbiting applications, but of greater concern for scan mirrors with little shielding from other materials, or for deep space missions that may encounter radiation fields many orders of magnitude higher than earth orbiting. SCHOTT is conducting a rigorous study [10] to provide data on radiation response of ZERODUR ${ }^{\circledR}$ into error budgets without large extrapolations of prior studies. ZERODUR ${ }^{\circledR}$ has been used without measurable change for over 40 years in space including on two NASA Great Observatories, all mirrors on Chandra and Secondary Mirror on Hubble.

\section{Recent augmentation of CNC machining of ZERODUR ${ }^{\circledR}$ at SCHOTT:}

In response to increasing demand for precision machined ZERODUR ${ }^{\circledR}$ for industrial and astronomical/surveilance requirements, including addressing requirements for monolithic mirrors of sizes in excess of $4 \mathrm{~m}$ diameter, the special throughput demands of the ELT production, and emerging markets for spaceborne lightweighted mirrors, SCHOTT established a new $5000 \mathrm{~m}^{2}$ Mainz Manufacturing Center in 2019. This constitutes both an unparalleled enhancement in both capacity and capabilities to precision machine ZERODUR ${ }^{\circledR}$. Figure 2 shows this facility overview. It features a vibration damped, temperature-controlled environment, capable of bringing a new level of accuracy to blank production. It is now feasible to address not only the mechanical features of lightweight mirrors, but also presents the accuracy to generate aspheric forms on the optical surface, including

a) Symmetric conic section forms

b) General symmetric aspheric forms

c) Off axis sections of aspheric forms

d) Freeform surfaces

This presents a deterministic and much faster way to generate such surfaces on optics and is especially important when the aspheric departure from the nearest sphere becomes large, as typical of many contemporary designs.

\section{Manufacturing Center since 2019}

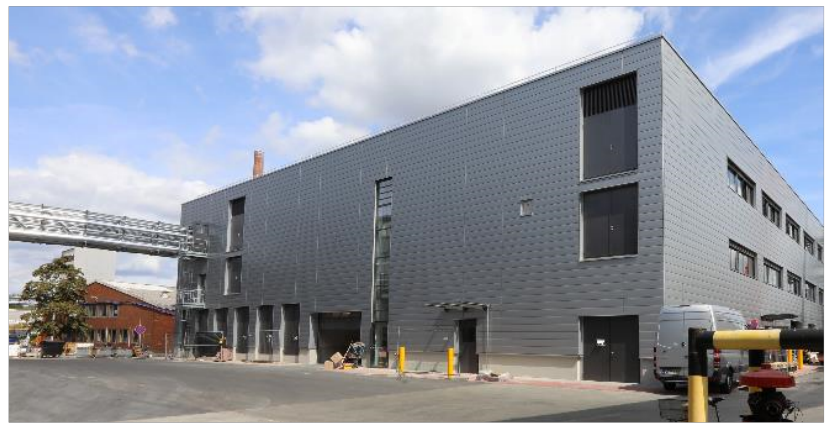

\begin{tabular}{|ll|}
\hline - Area & $\sim 5.000 \mathrm{~m}^{2}$ \\
- T stabilization & $\pm 1 \mathrm{~K}$ \\
- Controlled environment: & vibration, temperature \\
- Up to 14 CNC machines $(<$ & $4.5 \mathrm{~m}$ diameter $)$ \\
\hline
\end{tabular}

Large 3D CMM ZEISS MMZ-G since 2020

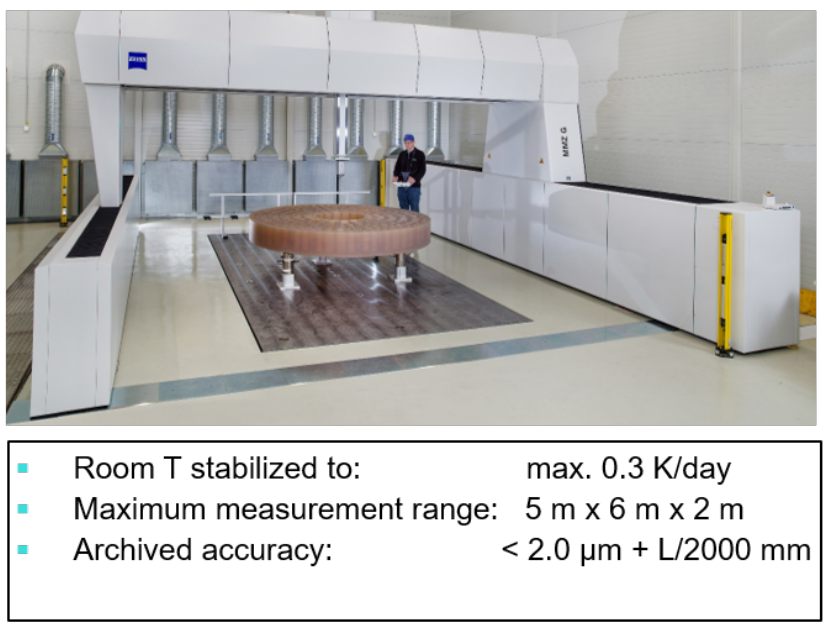

Figure 2: SCHOTT has added major new facility for machining and dimensional measurement of ZERODUR ${ }^{\circledR}$, providing not only capacity, but extending the size of machining to the largest blanks presently available, and introducing new regimes of accuracy in machining. We will later discuss how these capabilities, together with improvements of machining methods, introduces new horizons 
to mirror manufacture, even influencing the ease and lapse time in subsequent optical finishing, and potentially even supporting more efficient assembly and alignment.

Figure 2 represents the accredited accuracy of the new large 3-D coordinate measuring machine. For optical fabricators with deterministic small tool polishing, it is now feasible to go directly to this process without "restraining" the part with large tools.

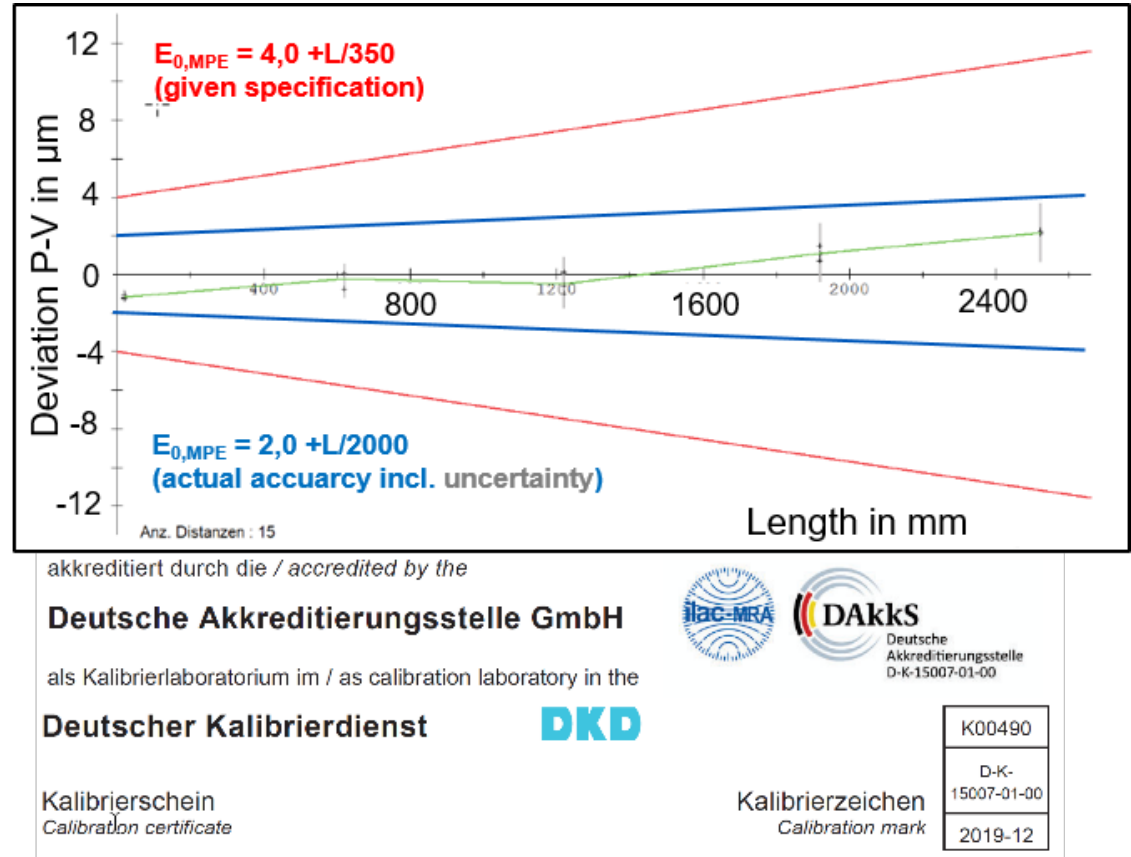

Figure 3: Highly accurate physical measurements of machined ZERODUR ${ }^{\circledR}$ are regularly made with SCHOTT's new metrology capability. For some optical fabricators, it is now feasible to directly go to deterministic small tool polishing. The efficiencies offered via this will be described in the next section.

Recent experience concerning the accuracy of SCHOTT machined parts is expressed in Figure 4. Methods of continuous improvement is part of standard practice.

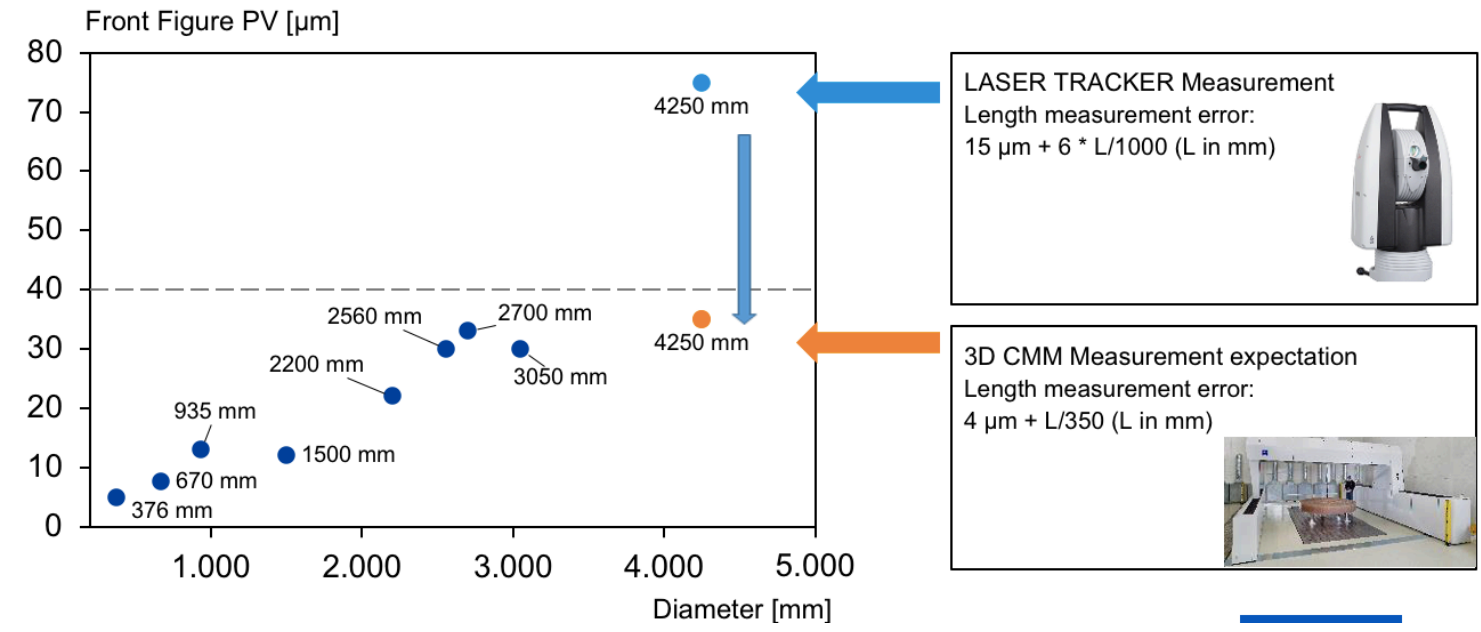

Figure 4: Recent experience in machining shows that errors are quite small as measured. This chart was made prior to the installation and qualification of the new large 3D coordinate measuring machine described above. The $4250 \mathrm{~mm}$ diameter part was measured with a laser tracker, exhibiting spurious azimuthal cumulative error. From the trend of the laser tracker error, we are confident that the error on this large part was no greater than $35 \mu \mathrm{m}$ p-v. 


\section{POTENTIAL EFFICIENCIES WITH SCHEDULE AND COST IMPLICATIONS}

Increasingly, cost becoming a critical variable in implementing spaceborne telescopes [11,12], especially for implementing constellations of dozens to hundreds of moderate size telescopes, typically less than $1 \mathrm{~m}$ in aperture and often of the order of $0.5 \mathrm{~m}$ aperture. The practice of Design-To-Cost (DTC) is becoming a guiding principle for such spaceborne systems. Lowest cost of a telescope is seldom achieved by using the lowest cost elements and manufacturers, but rather by a systematic look at how each architecture decision affects everything else.

Design-To-Cost may put increasing demand on the improved precision preparing the mirror blank's reflective surface, including generating the aspheric form of the mirror, while managing subsurface damage. With the new facility described above, excellent conformity of the mirror's reflective surface to the optical prescription can be generated using CNC mills and fixed abrasive tools. And this fixed abrasive generation is substantially more efficient and deterministic than that typically associated with loose abrasive grinding in an optical shop.

This process recognizes the special dilemma of the subsequent optical shop for polishing spaceborne mirror substrates, typically lightweighted. Both:
A. Form Errors
B. Sub-Surface Damage (SSD)

Are present. If there is a large volume of material to be removed because of A and B, large tools and large pressure must be applied to have reasonable durations of optical finishing. The consequence of this is both

I. Subsurface printing (also known as quilting) create a pattern at mid-spatial frequencies (MSF) across the mirror

II. Zonal errors are also induced from larger tools since the local radius of curvature varies across an aspheric surface

MSF errors across the pupil, both those caused by I. and II. do make a significant contribution to contrast degradation of the image [13]. MSF artifacts must be removed. The dilemma is that the optical shop in the past must induce MSF errors, and spend a substantial fraction of the optical finishing period of performance removing these errors, induced by necessary large tool work, with small tools and deterministic small volume polishing. In the sense of LEAN processes, this is wasteful.

With either fixed or loose abrasive grinding, a layer of SSD remains after each tool pass, and this depth relates to the abrasive grit size and other tribological matters [14]. In many cases, both surface accuracy and sub-surface damage can be controlled during SCHOTT deterministic machining to the degree that the optical manufacturer does not need to regenerate the surface with large tools and in the process inducing substructure print through, but rather go directly to small tools. Figure 5 schematically summarizes the DTC savings of overall time and effort that may be realized by having mirrors prepared in this manner. 


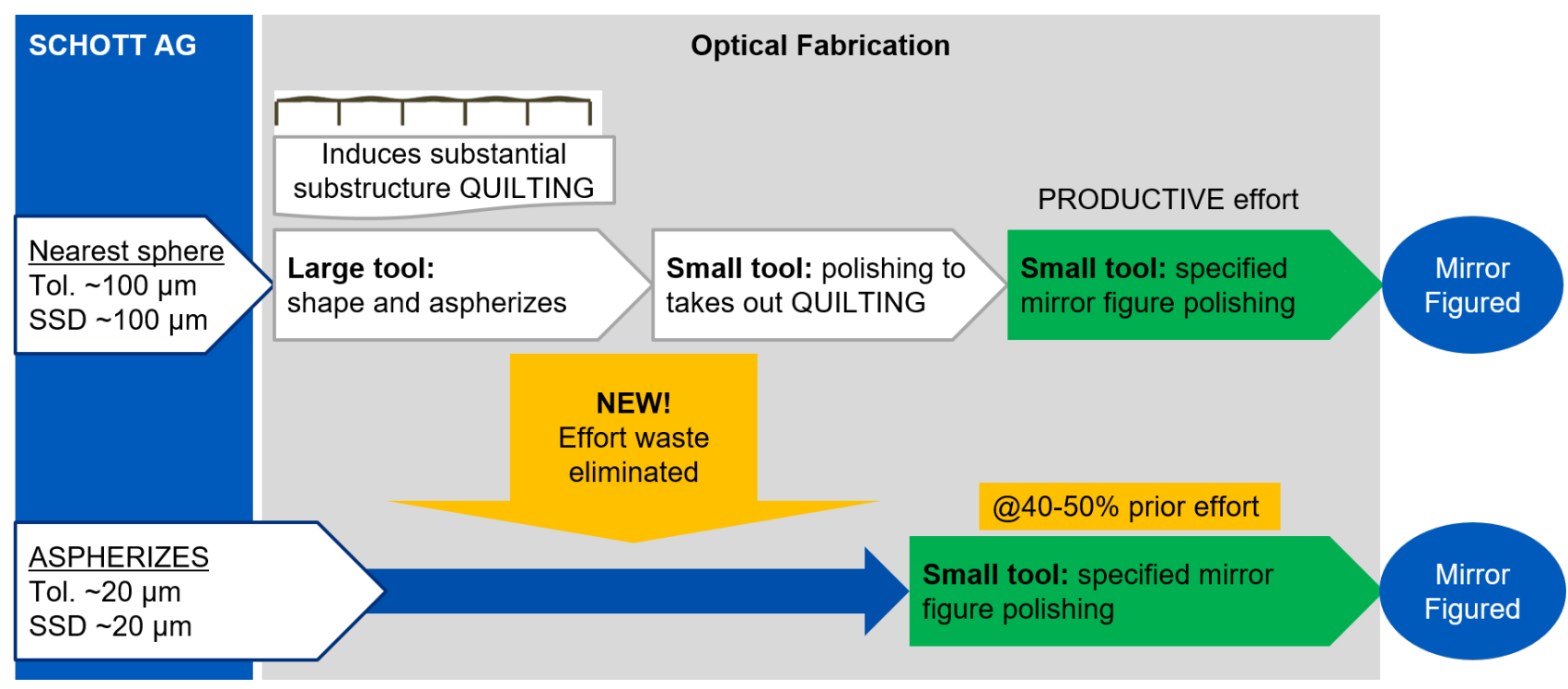

Figure 5: Schematic of potential realization of DTC savings from precision generation of ZERODUR ${ }^{\circledR}$ mirror blanks employing the efficiency of fixed abrasive generation of the optical surface, avoiding subsequent wasted effort in optical processing. This new paradigm may save up to $50 \%$ of the time in optical finishing.

\section{SUMMARY: PERFORMANCE OF SPACEBORNE MIRROR MATERIALS}

Material selection, and associated processes, is often one of the earliest decisions in the design process, and the effects resulting from this choice propagates throughout the production and operation of the assembly. This choice of mirror material invariably impacts the performance, complexity, risk and cost figures-of-merit of the optical system.

However, there are crucial factors in material processing to be considered for any material being evaluated:

- Is there a path toward sufficiently lightweighting the material, employing an established controlled process?

- Is the process for lightweighting efficient in cost and schedule?

- Do the processes to make the mirror offer space heritage for the wavelength and application, and associated high Technology Readiness Level

SCHOTT's processing of ZERODUR ${ }^{\circledR}$, evoking

a) Efficiently produced lightweighting by $\mathrm{CNC}$ machining

b) Accurate aspheric surfaces

c) Reduced SSD

d) Minimum dimensional response due to thermal perturbations

Together, these contribute to Better, Faster and Cheaper lightweight mirrors, and with the associated spaceborne telescopes, achieving more of the required performance with passive thermal means.

Thus, both the materials, and processes must be aligned to realize efficient high-performance implementation of costeffective spaceborne telescopes. Needs are addressed for monolithic mirrors in the size range from centimeters to over 4 meters in diameter, and in unit production, or production line deliveries. The telescope designer should consider if there is sufficient data available on each material being considered, and understand the processing used to make the material into 
mirror blanks. The parameters for mirror material selection are numerous, and failure to recognize any of these will have implications on performance, risk, complexity and ultimately cost of a system.

\section{ACKNOWLEDGEMENTS}

We wish to thank many technical people at SCHOTT for computational support and technical discussions. These include A Leys and Dr. P. Hartmann. We also thank Dr. Phil Stahl of NASA for his interest in ZERODUR ${ }^{\circledR}$, and his support in undertaking substantial NASA tests of lightweighted ZERODUR ${ }^{\circledR}$ mirror blanks.

\section{REFERENCES}

[1] Hull, T, J. Krieg, R. Jedamzik, T. Westerhoff "Material attributes that define performance and efficiency of spaceborne mirrors", Proc. ICSO 2020-203 (2021)

[2] Brooks, T, R. Eng, T. Hull, P. Stahl "Modeling the Extremely Lightweight ZERODUR ${ }^{\circledR}$ Mirror (ELZM) thermal soak test" Proc. SPIE 10374 (2017)

[3] Dohring, T., P. Hartmann, F-T. Lentes, R. Jedamzik, M. Davis. "Heritage of ZERODUR ${ }^{\circledR}$ glass ceramic for space applications", Proc. SPIE. 7425, Optical Materials and Structures Technologies IV (2009)

[4] Hull, T, T. Westerhoff "Extreme lightweight ZERODUR ${ }^{\circledR}$ mirrors: supporting characteristics for spaceborne applications", Proc. SPIE. 9143, Space Telescopes and Instrumentation (2014)

[5] Westerhoff, T., T. Hull, R. Jedamzik “ZERODUR ${ }^{\circledR}$ manufacturing capacity:ELT and more”, Proc. SPIE. 11116 (2020)

[6] Westerhoff, T., T. Hull, R. Jedamzik "Establishing a substrate manufacturing center for ZERODUR ${ }^{\circledR}$ 4-meter diameter lightweight mirrors", Proc. SPIE 11117 (2020)

[7] Jedamzik, R., T. Werner, T. Westerhoff "Production of the $4.26 \mathrm{~m}$ ZERODUR ${ }^{\circledR}$ mirror blank for the Advanced Technology Solar Telescope” Proc. SPIE 9151 (2014)

[8] Jedamzik, R., T. Westerhoff "Homogeneity of the coefficient of linear thermal expansion of ZERODUR ${ }^{\circledR}$ : a review of a decade of evaluations", Proc. SPIE 10401 (2017)

[9] Cohen, E and T. Hull, "Selection of a mirror technology for the 1.8-m Terrestrial Planet Finder demonstrator mission", Proc. SPIE 5494 (2004)

[10] Carré, A., T. Westerhoff, T. Hull, "Impact of ionizing radiations on ZERODUR ${ }^{\mathbb{}}$ ” Proc. SPIE 10698 (2018)

[11] Hull, T., T. Westerhoff "Lightweight ZERODUR ${ }^{\circledR}$ mirror blanks: recent advances supporting faster, cheaper, and better spaceborne optical telescope assemblies" Proc. SPIE 9241 (2014)

[12] A. Leys, T. Hull, T. Westerhoff "Production of ELZM mirrors: performance coupled with attractive schedule, cost, and risk factors", Proc. SPIE 9911 (2016)

[13] Hull, T., Riso, M., Barentine, J., Magruder, A. "Mid-spatial frequencies matters: examples of the control of the power spectral density and what that means to the performance of imaging systems", Proc. SPIE 8353 (2012)

[14] A. Leys, T. Hull, A. Carre, T. Westerhoff, "Correlation of subsurface damage and surface roughness on ZERODUR ${ }^{\circledR}$ ground surfaces by using rotation table peripheral grinding process" ICSO 2020-250 (2021)

[15] Hull, T. et al. "Game-changing approaches to affordable advanced lightweight mirrors: Extreme ZERODUR ${ }^{\circledR}$ lightweighting and relief from the classical polishing parameter constraint” Proc. SPIE 8125 (2011)

[16] Hull, T. et al. "Game-changing approaches to affordable advanced lightweight mirrors II: Extreme ZERODUR ${ }^{\circledR}$ lightweighting and relief from the classical polishing parameter constraint” Proc. SPIE 8450 (2012) 